\title{
Exchange-spring behavior in nanopowders of $\mathrm{CoFe}_{2} \mathrm{O}_{4}-\mathrm{CoFe}_{2}$
}

J. M. Soares, F. A. O. Cabral, J. H. de Araújo, and F. L. A. Machado

Citation: Appl. Phys. Lett. 98, 072502 (2011); doi: 10.1063/1.3552677

View online: https://doi.org/10.1063/1.3552677

View Table of Contents: http://aip.scitation.org/toc/apl/98/7

Published by the American Institute of Physics

\section{Articles you may be interested in}

Enhancement of $(B H)_{\text {max }}$ in a hard-soft-ferrite nanocomposite using exchange spring mechanism Journal of Applied Physics 106, 073902 (2009); 10.1063/1.3213341

Exchange spring behaviour in $\mathrm{SrFe}_{12} \mathrm{O}_{19}-\mathrm{CoFe}_{2} \mathrm{O}_{4}$ nanocomposites

AIP Advances 5, 077137 (2015); 10.1063/1.4927150

On the origin of remanence enhancement in exchange-uncoupled $\mathrm{CoFe}_{2} \mathrm{O}_{4}$-based composites

Applied Physics Letters 105, 202405 (2014); 10.1063/1.4902351

Size selected synthesis of $\mathrm{CoFe}_{2} \mathrm{O}_{4}$ nanoparticles prepared in a chitosan matrix Journal of Applied Physics 107, 09A746 (2010); 10.1063/1.3339784

Diversity of the magnetic coupling behaviors in the $\mathrm{CoFe}_{2} / \mathrm{CoFe}_{2} \mathrm{O}_{4}$ system Applied Physics Letters 86, 192514 (2005); 10.1063/1.1923763

Static and dynamic magnetic properties of spherical magnetite nanoparticles Journal of Applied Physics 94, 3520 (2003); 10.1063/1.1599959

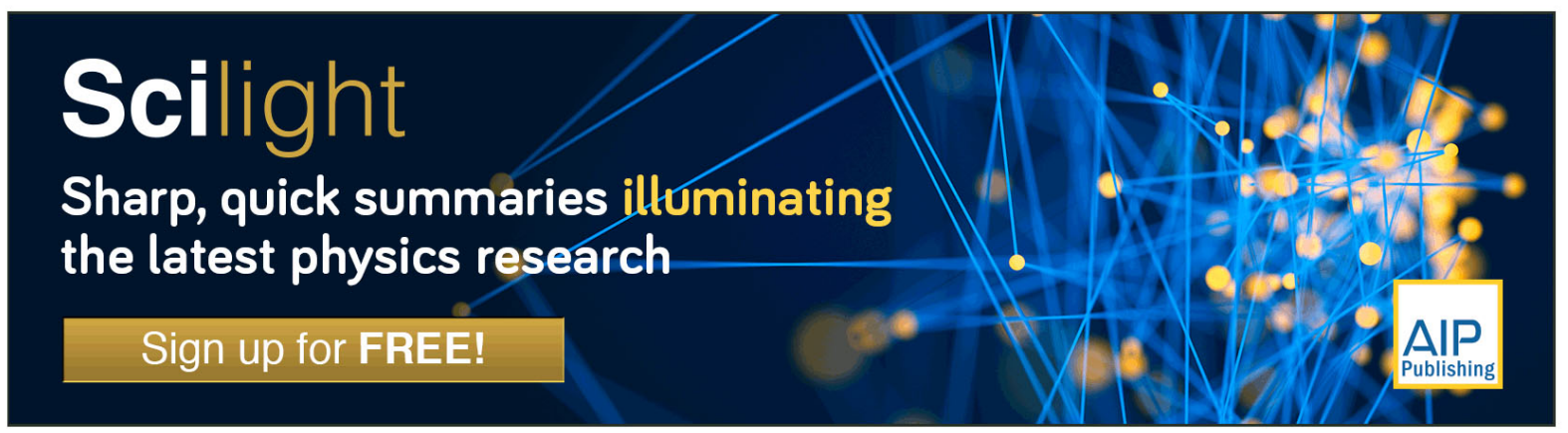




\title{
Exchange-spring behavior in nanopowders of $\mathrm{CoFe}_{2} \mathrm{O}_{4}-\mathrm{CoFe}_{2}$
}

\author{
J. M. Soares, ${ }^{1, a)}$ F. A. O. Cabral, J. H. de Araújo, ${ }^{2}$ and F. L. A. Machado ${ }^{3}$ \\ ${ }^{1}$ Departamento de Física, Universidade do Estado do Rio Grande do Norte, 59610-010 Mossoró, \\ Rio Grande do Norte, Brazil \\ ${ }^{2}$ Departamento de Física Teórica e Experimental, Universidade Federal do Rio Grande do Norte, \\ 59072-970 Natal, Rio Grande do Norte, Brazil \\ ${ }^{3}$ Departamento de Física, Universidade Federal de Pernambuco, 50670-901 Recife, Pernambuco, Brazil
}

(Received 22 December 2010; accepted 12 January 2011; published online 15 February 2011)

\begin{abstract}
The nature of the magnetic interactions among core-shell nanoparticles of $\mathrm{CoFe}_{2} \mathrm{O}_{4}-\mathrm{CoFe}_{2}$ having an average particle size of $32 \mathrm{~nm}$ were investigated by $\delta m$ plots. It was found that the interactions are mainly demagnetizing in nature for nanoparticles with core diameters larger than $26 \mathrm{~nm}$. For smaller values of core diameters, the exchange-spring phenomenon takes place due to the exchange-coupling at the core-shell interface. The critical thickness of the shell was estimated to be $7.8 \mathrm{~nm}$. The overall results are in excellent agreement with current theoretical models. (C) 2011 American Institute of Physics. [doi:10.1063/1.3552677]
\end{abstract}

The need for improving some magnetic properties using magnetic core-shell nanoparticles has inspired an area of great scientific and technological interest. ${ }^{1-10}$ Exchangecoupling through the core-shell interface was found to drastically modify the magnetic properties of nanocomposite combining the high magnetization of a soft-magnetic phase with the high anisotropy of a hard one. Basically, the magnetic fields required to perform a reversible rotation of the magnetic moments in the soft phase are defined by the anisotropy field of the hard magnetic phase and this was named exchange-spring phenomenon.

In this work, the nature of the magnetic interactions in magnetic core-shell nanopowders, composed of a highly anisotropic ferrite $\left(\mathrm{CoFe}_{2} \mathrm{O}_{4}\right)$ and a soft-ferromagnetic phase $\left(\mathrm{CoFe}_{2}\right)$, was investigated. The demagnetizing remanence curves allowed us to determine the distribution of critical fields. Moreover, the dependence of the critical field with the thickness of the soft-magnetic phase yielded two well defined regimes. From them, we determined the critical thickness of the shell required for optimizing the exchange-spring effect in these nanocomposite materials.

$\mathrm{CoFe}_{2} \mathrm{O}_{4}-\mathrm{CoFe}_{2}$ core-shell nanoparticles were obtained from a single-phase $\mathrm{CoFe}_{2} \mathrm{O}_{4}$ powder prepared by an ionic coordination technique. ${ }^{11}$ The average particle size for the $\mathrm{CoFe}_{2} \mathrm{O}_{4}$ powder was obtained by using $\mathrm{x}$-ray diffraction (XRD) microscopy and it was found to be $32 \mathrm{~nm}$. $\mathrm{CoFe}_{2}$ shells with different thicknesses were readily obtained by reducing $\mathrm{CoFe}_{2} \mathrm{O}_{4}$ powder samples under a controlled hydrogen flux for various period of time $(15,30,60,90$, and 120 $\min )$ at $300{ }^{\circ} \mathrm{C}$. The ferromagnetic iron-cobalt shell is actually formed when the oxygen atoms are removed from the iron-cobalt ferrite core. This procedure ensures that the exchange interaction among the spins of the soft and hard magnetic phases occur within distances of the order of the lattice parameters. The reduction reaction occurring in the cobalt ferrite is $\mathrm{CoFe}_{2} \mathrm{O}_{4}+4 \mathrm{H}_{2} \rightarrow \mathrm{CoFe}_{2}+4 \mathrm{H}_{2} \mathrm{O}$. Assuming that the particles are spherical, one can calculate the thickness of both core and shell by applying the Scherrer formula to the most intense peak in the XRD data for the $\mathrm{CoFe}_{2} \mathrm{O}_{4}$ phase.

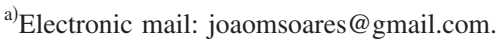

Assuming that the particles are spherical, the thickness of the $\mathrm{CoFe}_{2}$ shell can be readily estimated, e.g., $\delta_{\mathrm{CF}}=\left(d_{0}\right.$ $\left.-d_{\mathrm{CFO}}\right) / 2$, where $d_{0}$ is the average particle size of the starting ferrite and $d_{\mathrm{CFO}}$ is the average particle size of the ferrite phase after being reduced (core). Table I shows the values of $d_{\mathrm{CFO}}$ obtained for the series of samples used in the present work.

Typical x-ray diffractograms are shown in Fig. 1(a) for powder samples of $\mathrm{CoFe}_{2} \mathrm{O}_{4}$ and for samples $\mathrm{E}$ and $\mathrm{C}$. It is important to mention that we were able to index all the peaks to the $\mathrm{CoFe}_{2} \mathrm{O}_{4}$ and $\mathrm{CoFe}_{2}$ phases. This indicates that metallic diffusion may not be an important process while the reduction reaction is taking place. The somewhat low temperature $\left(300{ }^{\circ} \mathrm{C}\right)$ used to perform the reaction may be one of the reasons for this. Significant metallic diffusion, if present, would lead to the formation of a solid solution which, in turn, would be seen by XRD or it would appear in the magnetization data as a decoupled magnetic contribution. Figure 1(b) shows a transmission electron microscopy (TEM) image for the $\mathrm{CoFe}_{2} \mathrm{O}_{4}$ sample. A particle size histogram [Fig. 1(c)] based on the micrograph shows a narrow distribution with an average particle size of $31.8 \mathrm{~nm}$ which, in turn, is in good agreement with the one $(32.0 \mathrm{~nm})$ obtained by XRD. A TEM image for a nanoparticle of sample B is shown in Fig. 1(d). There, one may note a core-shell structure where the core diameter is about $24 \mathrm{~nm}$. This value is also in good agreement with the one obtained by $\operatorname{XRD}\left(d_{\mathrm{CFO}}=25.8 \mathrm{~nm}\right)$. Samples of the pure and of the reduced nanopowders were compressed in cylindrical glass sample holders and placed in

TABLE I. Average core $\left(\mathrm{CoFe}_{2} \mathrm{O}_{4}\right)$ diameter $d_{\mathrm{CFO}}$, thickness of the shell $\left(\mathrm{CoFe}_{2}\right) \delta_{\mathrm{CF}}$, coercive field $H_{c}$, and saturation $M_{S}$ and remanence $M_{r}$ magnetizations.

\begin{tabular}{lccccc}
\hline \hline Sample & $\begin{array}{c}d_{\mathrm{CFO}} \\
(\mathrm{nm})\end{array}$ & $\begin{array}{c}\delta_{\mathrm{CF}} \\
(\mathrm{nm})\end{array}$ & $\begin{array}{c}H_{c} \\
(\mathrm{kOe})\end{array}$ & $\begin{array}{c}M_{S} \\
(\mathrm{emu} / \mathrm{g})\end{array}$ & $\begin{array}{c}M_{r} \\
(\mathrm{emu} / \mathrm{g})\end{array}$ \\
\hline A & 30.3 & 1.2 & 1.08 & 73.8 & 29.1 \\
$\mathrm{~B}$ & 25.8 & 3.4 & 0.92 & 79.5 & 27.8 \\
$\mathrm{C}$ & 15.2 & 8.7 & 0.83 & 103.0 & 29.0 \\
$\mathrm{D}$ & 13.6 & 9.5 & 0.59 & 137.8 & 31.0 \\
$\mathrm{E}$ & 10.0 & 11.3 & 0.54 & 184.3 & 49.4 \\
\hline \hline
\end{tabular}



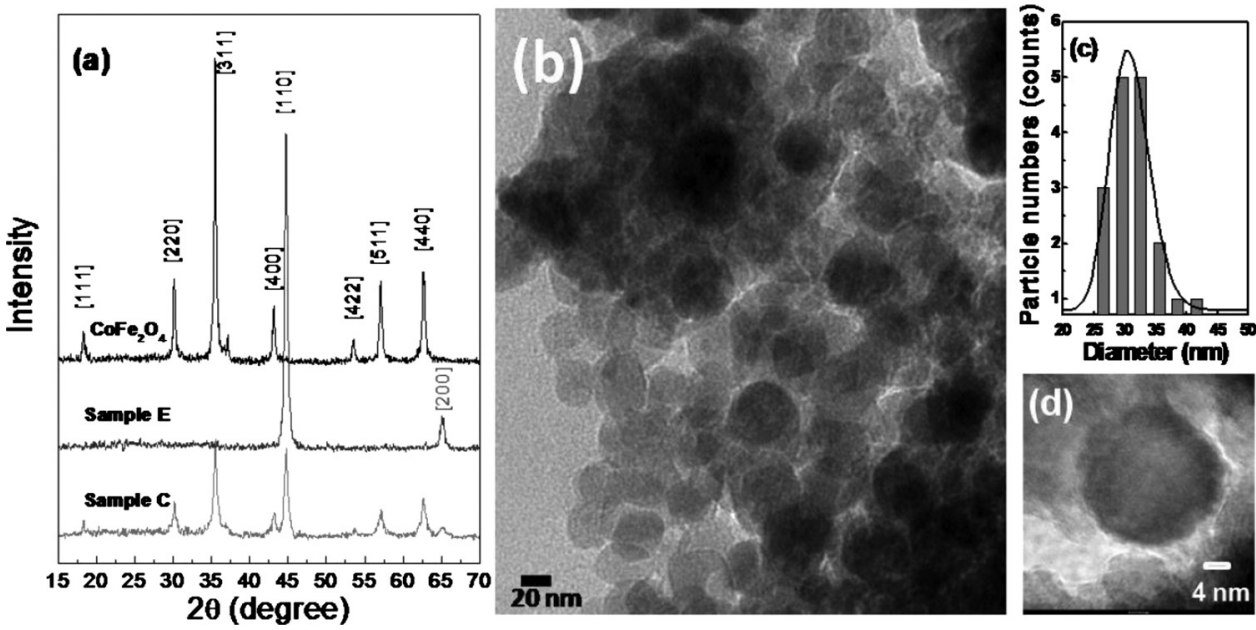

FIG. 1. (a) X-ray diffractograms for pure $\mathrm{CoFe}_{2} \mathrm{O}_{4}$ and for samples $\mathrm{E}$ and C. (b) TEM image for a $\mathrm{CoFe}_{2} \mathrm{O}_{4}$ powder sample. (c) Particle size histogram for sample B. (d) High resolution TEM image for a sample B nanoparticle. a vibrant sample magnetometer for room temperature magnetization measurements. The randomly packed polycrystalline grains form a random anisotropy nanoparticulated system. Hysteresis loops obtained for the $\mathrm{CoFe}_{2} \mathrm{O}_{4}$ sample yielded a coercivity of $1.32 \mathrm{kOe}$ and a saturation $\left(M_{S}\right)$ and a remanent $\left(M_{r}\right)$ magnetization of 68.0 and $33.7 \mathrm{emu} / \mathrm{g}$, respectively. The $M_{r} / M_{S}$ ratio was found to be close to 0.5 , which is the appropriated value for coherent magnetization rotation to occur in single-domain nanoparticles.

The $\delta m$ curves were built using the magnetizing $M_{r}(H)$ and demagnetizing $M_{d}(H)$ remanent magnetizations measured as follows. First, the isothermal remanent magnetization $M_{r}(H)$ is measured after a magnetic field $H(H>0)$ is applied and removed. This procedure is repeated with the magnitude of $H$ being gradually increased until the magnetization reaches saturation $\left(H=H_{\text {sat }}\right)$. Next, the remanent demagnetizing magnetization is obtained following a somewhat similar procedure. Starting from the maximum remanence, e.g., after the sample has been saturated and $H_{\text {sat }}$ has been removed, one applies and removes a negative field $(H<0)$ and measures the remanence. $H$ is then gradually increased toward (negative) saturation. It is important to use the same values of $H$ in both procedures to allow a good correlation between both remanent magnetization data. Finally, $M_{r}(H)$ and $M_{d}(H)$ are normalized by the saturation remanence $M_{r}\left(H_{\text {sat }}\right): m_{r}=M_{r}(H) / M_{r}\left(H_{\text {sat }}\right)$ and $m_{d}=M_{d}(H) / M_{d}\left(H_{\text {sat }}\right)$.

Figure 2 shows a graph of $m_{d}$ as a function of $H$ for each of the core-shell samples investigated in the present work.

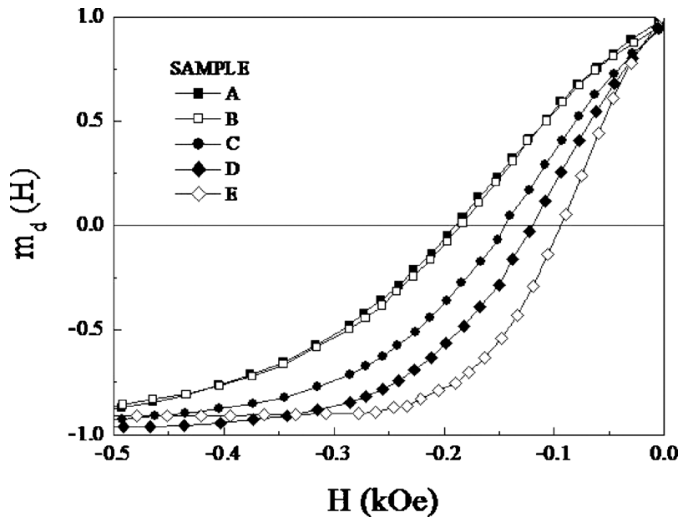

FIG. 2. Demagnetizing remanence vs $H$.
The behavior of $m_{d}$ for samples A and B follows the same trends. However, the curvature in $M_{d}(H)$ increases substantially for the core-shell samples with larger values of $\delta_{\mathrm{CF}}$. This is a clear indication that the mechanisms governing the magnetization process is strongly influenced by the coreshell structure. The nature of the interactions in a set of magnetic nanoparticles has been determined by using the Wohlfarth relationship established for noninteracting singledomain uniaxial nanoparticles, $m_{d}=1-2 m_{r}{ }^{12}$ A deviation from the Wohlfarth relationship is an indication that the nanoparticles are interacting among themselves. The deviation is better seen through a relationship known as $\delta m$ and defined as $\delta m=m_{d}(H)-\left[1-2 m_{r}(H)\right]$. $^{13}$

The inset in Fig. 3 shows the $\delta m$ plots for our samples. Note that the magnitude of $\delta m$ is negative for samples A and $\mathrm{B}$, while part of the curves are positive for samples $\mathrm{C}-\mathrm{E}$. A similar behavior has been observed in some metallic alloys. ${ }^{3,14}$ Furthermore, it was found that $\delta m$ becomes less negative when the core-shell ratio is reduced. The negative peak in $\delta m$ is a signature of demagnetizing (dipolar) interactions in nanopowder samples. ${ }^{15}$ The observed reduction in the size of the negative peak may be interpreted as being due to the presence of magnetizing (exchange-type) interactions. Moreover, its influence in the size of the demagnetizing peak can be made more explicit by subtracting the $\delta m$ curve for

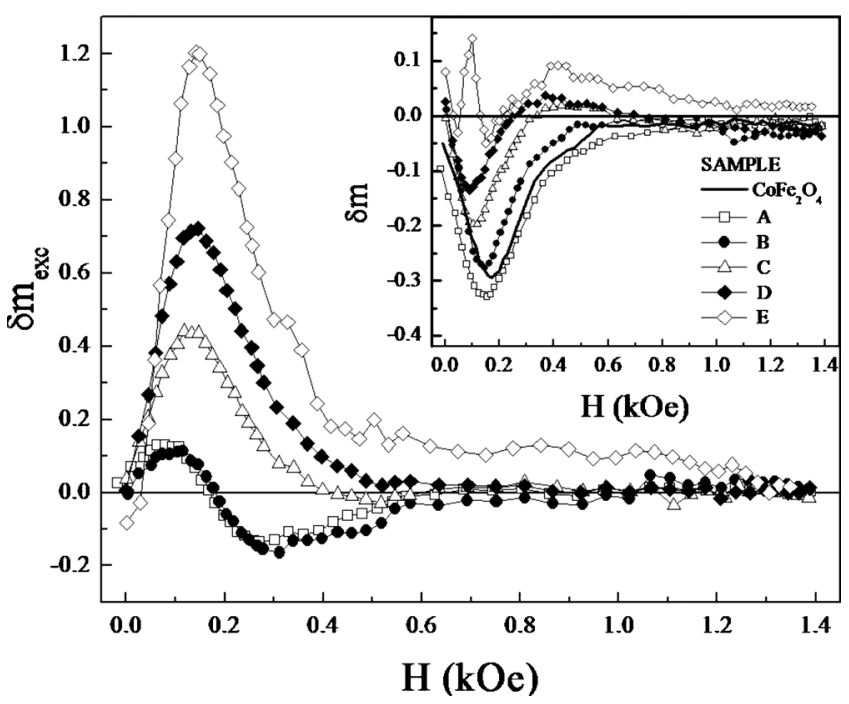

FIG. 3. $\delta m_{\mathrm{exc}}$ and $\delta m$ (inset) plots. 


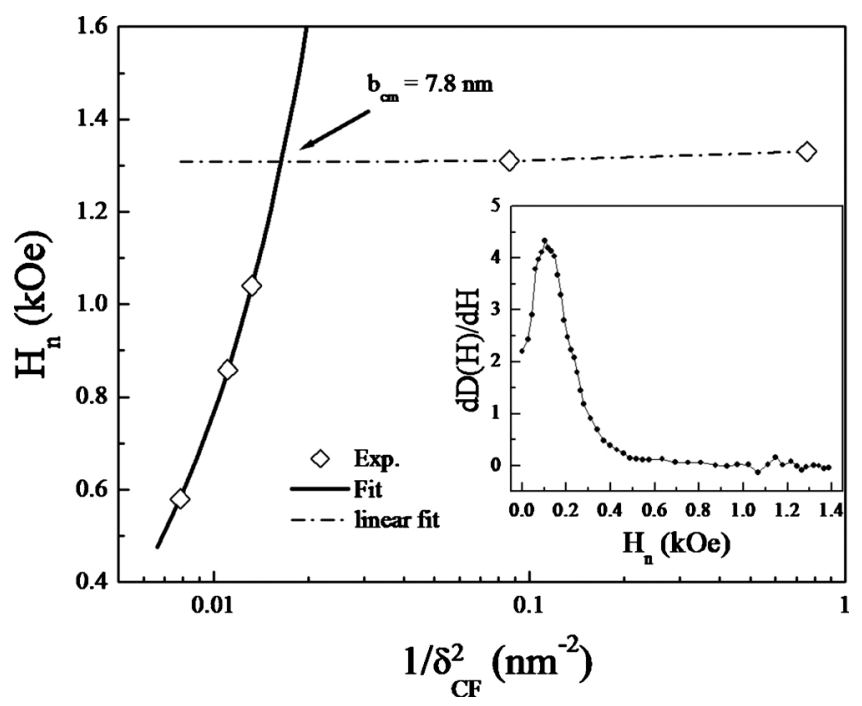

FIG. 4. Nucleation field vs the inverse of the square of the shell thickness; open diamonds are experimental points. The solid line is a fit using a model presented in the main text and the dashed line is a guide to the eyes. The inset is the distribution of critical fields $\left(H_{n}\right)$ for sample $\mathrm{C}$.

$\mathrm{CoFe}_{2} \mathrm{O}_{4}$ from the $\delta m$ curve for the $\mathrm{CoFe}_{2} \mathrm{O}_{4}-\mathrm{CoFe}_{2}$ coreshell samples: $\delta m_{\mathrm{exc}}=\left[m_{d \mathrm{CFO}}(H)-m_{d}(H)\right]+2\left[m_{r \text { CFO }}(H)\right.$ $\left.-m_{r}(H)\right]$, where $m_{r \mathrm{CFO}}$ and $m_{d \mathrm{CFO}}$ are the magnetizing and demagnetizing remanences, respectively, for the cobalt ferrite powder sample. The $\delta m_{\text {exc }}$ plots for the core-shell samples are shown in the main part of Fig. 3. There, one may note that fully positive peaks are only observed in the data for samples C-E, while nearly half of the data for samples A and $\mathrm{B}$ is negative. ${ }^{16}$ The intensity of peaks rapidly increases when the thickness of the $\mathrm{CoFe}_{2}$ shell is also increased. The source of exchange-coupling is mainly twofold: from interactions occurring at the interface of the core-shell and from the contact between nanoparticles. The magnetic field required for nucleating irreversible magnetization reversal $\left(H_{n}\right)$ is a signature for exchange-spring magnets. It can be estimated from the anisotropy constant $K_{k}$ of the hard magnetic phase and from the saturation magnetization of the soft one: ${ }^{17} H_{n}=K_{k} / M_{S m}$. Experimentally, the nucleation field may be obtained from the magnetic field dependence of the demagnetizing remanence $M_{d}(H)$ by defining the quantity $D(H)=\left[M_{r}-M_{d}(H)\right] / 2 M_{r}$. The maximum in the derivative of $D(H)$ with respect to $H$ yields a value for $H_{n}$. The inset in Fig. 4 is a plot of $d D(H) / d H$ versus $H$ for sample C. Thus, the maximum of $d D(H) / d H$ yields $H_{n}=1040$ Oe for sample C. This analysis was also applied for the other samples and the corresponding values for $H_{n}$ were plotted as a function of the inverse of the square of the shell thickness $\delta_{\mathrm{CF}}$ in the main part of Fig. 4. Kneller and Hawig ${ }^{17}$ have shown that exchange-spring magnets may have their magnetic properties optimized when $H_{n}$ is related to a critical shell thickness $b_{m}$ by $H_{n}=\pi^{2} A_{m} / 2 M_{S m} b_{m}^{2}$, where $A_{m}$ is the exchange constant of the magnetically soft phase. In Fig. 4, one can observe two well defined regimes for the nucleation fields. For small values of $\delta_{\mathrm{CF}}$, the nucleation field is nearly constant $\left(H_{n}\right.$ $\cong 1300 \mathrm{Oe}$ ). In this regime, the $\mathrm{CoFe}_{2} \mathrm{O}_{4}$ phase (core) is decoupled from the $\mathrm{CoFe}_{2}$ shell and the magnetizing process is reversible. In the second regime, both phases couple to- gether through exchange-coupling and the nucleation field decreases with increasing values of $\delta_{\mathrm{CF}}$. The full line in Fig. 4 is a best fit of $H_{n}$, while the dashed line is a guide to the eyes. $b_{m}$ may be determined by taking the value of $\delta_{\mathrm{CF}}$ at the crossing over point between the two regimes established by the dependence of $H_{n}$ with $\delta_{\mathrm{CF}}$. For our samples, a value of $7.8 \mathrm{~nm}$ for $b_{m}$ was found. Furthermore, the fitting yielded $8.5 \times 10^{-10} \mathrm{Oe} / \mathrm{cm}^{2}$ for the coefficient $\pi^{2} A_{m} / 2 M_{S m}$. Now, using $M_{S m}=1850 \mathrm{emu} / \mathrm{cm}^{3}$ (Ref. 18) for the saturation magnetization of $\mathrm{CoFe}_{2}$ and the fitting parameter, one gets the exchange constant $A_{m}=3.2 \times 10^{-7} \mathrm{erg} / \mathrm{cm}$ for the soft phase. In addition, $A_{m}$ and $b_{c m}$ can now be used to calculate the anisotropy constant $K_{k}=2.1 \times 10^{6} \mathrm{erg} / \mathrm{cm}^{3}$ for the magnetically hard phase from $K_{k}=\pi^{2} A_{m} / 2 b_{m}^{2}$. The value obtained for $K_{k}$ is within the range accepted for cobalt ferrite. ${ }^{19}$

In conclusion, core-shell nanoparticles of $\mathrm{CoFe}_{2} \mathrm{O}_{4}-\mathrm{CoFe}_{2}$ with shell thickness ranging from 1.2 to $11.0 \mathrm{~nm}$ were prepared by reducing $\mathrm{CoFe}_{2} \mathrm{O}_{4}$ in hydrogen atmospheres. Their intraparticle and interparticle interactions were studied using $\delta m$ plots. The core-shell was found to couple through exchange-coupling and the optimum thickness parameter was estimated to be $7.8 \mathrm{~nm}$ using a current theoretical model for exchange-spring magnets. Other sample parameters were determined self-consistently using current theoretical models.

This work is partially supported by CNPq, CAPES, FAPERN, and FACEPE (Brazilian Agencies).

${ }^{1}$ V. Skumryev, S. Stoyanov, Y. Zhang, G. Hadjipanayis, D. Givord, and J. Nogués, Nature (London) 423, 850 (2003).

${ }^{2}$ H.-G. Boyen, G. Kstle, K. Zrn, T. Herzog, F. Weigl, P. Ziemann, O. Mayer, C. Jerome, M. Mller, P. Spatz, M. Garnier, and P. Oelhafen, Adv. Funct. Mater. 13, 359 (2003).

${ }^{3}$ H. Zeng, J. Li, Z. L. Wang, J. P. Liu, and S. Sun, Nano Lett. 4, 187 (2004).

${ }^{4}$ J. Li, H. Zeng, S. Sun, J. P. Liu, and Z. L. Wang, J. Phys. Chem. B 108, 14005 (2004).

${ }^{5}$ G. Hassnain Jaffari, S. Rizwan Ali, S. K. Hasanain, G. Güntherodt, and S. Ismat Shah, J. Appl. Phys. 108, 063921 (2010).

${ }^{6}$ J. Park, E. Kang, C. J. Bae, J.-G. Park, H.-J. Noh, J.-Y. Kim, J.-H. Park, H. M. Park, and T. Hyeon, J. Phys. Chem. B 108, 13594 (2004).

${ }^{7}$ J.-I. Park, M. G. Kim, Y. Jun, J. S. Lee, W.-R. Lee, and J. Cheon, J. Am. Chem. Soc. 126, 9072 (2004).

${ }^{8}$ S. A. Majetich and Y. Jin, Science 284, 470 (1999).

${ }^{9}$ I. Brigger, C. Dubernet, and P. Couvreur, Adv. Drug Delivery Rev. 54, 631 (2002).

${ }^{10}$ D. Portet, B. Denizot, E. Rump, J. Lejeune, and P. J. Jallet, J. Colloid Interface Sci. 238, 37 (2001).

${ }^{11}$ A. L. Gurgel, J. M. Soares, D. S. Chaves, D. S. Chaves, M. M. Xavier, Jr., M. A. Morales, and E. M. Baggio-Saitovitch, J. Appl. Phys. 107, 09A746 (2010)

${ }^{12}$ E. P. Wohlfarth, J. Appl. Phys. 29, 595 (1958).

${ }^{13}$ P. E. Kelly, K. O'Grady, P. I. Mayo, and R. W. Chantrell, IEEE Trans. Magn. 25, 3881 (1989)

${ }^{14}$ J. M. Soares, F. L. A. Machado, J. H. de Araújo, F. A. O. Cabral, and M F. Ginani, Phys. Rev. B 72, 184405 (2005).

${ }^{15}$ D. Bueno-Baqués, A. Medina-Boudrí, and J. Matutes-Aquino, J. Magn. Magn. Mater. 226-230, 1412 (2001).

${ }^{16}$ H. Zeng, S. Sun, T. S. Vedantam, J. P. Liu, Z. R. Dai, and Z. L. Wang, Appl. Phys. Lett. 80, 2583 (2002).

${ }^{17}$ E. F. Kneller and R. Hawig, IEEE Trans. Magn. 27, 3588 (1991).

${ }^{18}$ B. D. Cullity and C. D. Graham, Introduction to Magnetic Materials, 2nd ed. (John Wiley \& Sons, Inc., Hoboken, New Jersey, 2009).

${ }^{19}$ G. C. Papaefthymiou, S. R. Ahmed, and P. Kofinas, Rev. Adv. Mater. Sci. 10, 306 (2005). 